\title{
Hybrid Diamond/ Carbon Fiber Microelectrodes Enable Multimodal Electrical/Chemical Neural Interfacing.
}

Maryam A. Hejazi ${ }^{1}$, Wei Tong ${ }^{1,2,3}$, Alastair Stacey ${ }^{1}$, Artemio Soto-Breceda ${ }^{2,4,5}$, Michael R. Ibbotson ${ }^{2,3}$, Molis Yunzab $^{2}$, Matias I. Maturana ${ }^{6}$, Ali Almasi ${ }^{2}$, Young Jun Jung ${ }^{2,3}$, Shi Sun ${ }^{2,3}$, Hamish Meffin ${ }^{2}$, Jian Fang, ${ }^{7,8}$ Melanie E.M.Stamp ${ }^{1}$, Kumaravelu Ganesan ${ }^{1}$, Kate Fox ${ }^{9,10}$, Aaqil Rifai ${ }^{9}$, Athavan Nadarajah ${ }^{1}$, Samira Falahatdoost $^{1}$, Steven Prawer ${ }^{1}$, Nicholas V. Apollo ${ }^{1,11}$, David J. Garrett ${ }^{1 *}$.

1. School of Physics, University of Melbourne, Parkville, Victoria, Australia

2. National Vision Research Institute, Australian College of Optometry, Carlton, Victoria, Australia

3. Department of Optometry and Vision Sciences, University of Melbourne, Parkville, Victoria, Australia

4. Department of Biomedical Engineering, University of Melbourne, Parkville, Victoria, Australia

5. Data 61, CSIRO, Docklands, Victoria, Australia

6. Department of Medicine, St Vincent's Hospital, Melbourne, Australia

7. ARC Centre of Excellence for ElectroMaterials Science (ACES), Australia

8. Deakin University, Institute for Frontier Materials, Geelong, VIC 3216, Australia

9. School of Engineering, RMIT University, Melbourne, Victoria, Australia

10. Centre for Additive Manufacturing, RMIT University, PO Box 2476, Melbourne, Victoria, Australia

11. Center for Neural Engineering and Therapeutics, University of Pennsylvania, Philadelphia, PA, USA

*Corresponding Author: dgarrett@unimelb.edu.au

\begin{abstract}
Implantable medical devices are now in regular use to treat or ameliorate medical conditions, including movement disorders, chronic pain, cardiac arrhythmias, and hearing or vision loss. Aside from offering alternatives to pharmaceuticals, one major advantage of device therapy is the potential to monitor treatment efficacy, disease progression, and perhaps begin to uncover elusive mechanisms of diseases pathology. In an ideal system, neural stimulation, neural recording, and electrochemical sensing would be conducted by the same electrode in the same anatomical region. Carbon fiber $(\mathrm{CF})$ microelectrodes are the appropriate size to achieve this goal and have shown excellent performance, in vivo. Their electrochemical properties, however, are not suitable for neural stimulation and electrochemical sensing. Here, we present a method to deposit high surface area conducting diamond on CF microelectrodes. This unique hybrid microelectrode is capable of recording single-neuron action potentials, delivering effective electrical stimulation pulses, and exhibits excellent electrochemical dopamine detection. Such electrodes are needed for the next generation of miniaturized, closed-loop implants that can self-tune therapies by monitoring both electrophysiological and biochemical biomarkers.
\end{abstract}

Keyword: Neural interface, carbon fiber, diamond, neural stimulation, biosensing, recording.

\section{Introduction}

The cardiac pacemaker, cochlear implant, deep brain stimulator for Parkinson's disease and spinal cord stimulator for pain control are key examples of implantable devices that have progressed from basic research to commercial and clinical success. These examples represent only a small fraction of the possible clinical targets that could be addressed with "electric medicine". There are many other approaches under development, including electrical stimulation for epilepsy, psychiatric disorders, hypertension, heart failure, gastrointestinal disorders, type II diabetes, and inflammatory disorders [1]. Additionally, recording devices are under investigation for limb prosthetics towards the promise of mobility for amputees and those suffering from paralysis $[2,3]$. By directly using electrical impulses to modulate the body's neural circuits, electric medicine 
provides a new paradigm for the treatment of disease. If devices can be miniaturized and their performance improved, electric medicine could be applied to a much broader range of clinical targets.

There is strong interest in implantable neuromodulation technologies that not only treat conditions but also record the efficacy of treatment, i.e. they could remotely inform specialists, measure their own efficacy, selfcalibrate and react to the changing needs of the user. In the field, this is referred to as a closed-loop device, as illustrated in Figure 1. Current neural implants are typically comprised of electrodes that primarily perform one function: either record from or stimulate neurons. Electrodes that perform more than one function are one way that overall device invasiveness can be minimized. Miniaturization of electrodes to microwire geometries, not only enables fabrication of smaller devices but has been shown to lead to less tissue damage and lower electrode failure, particularly when using carbon materials $[4,5]$. At 7-10 $\mu \mathrm{m}$ in diameter CFs are similar in diameter to the soma of individual neurons.

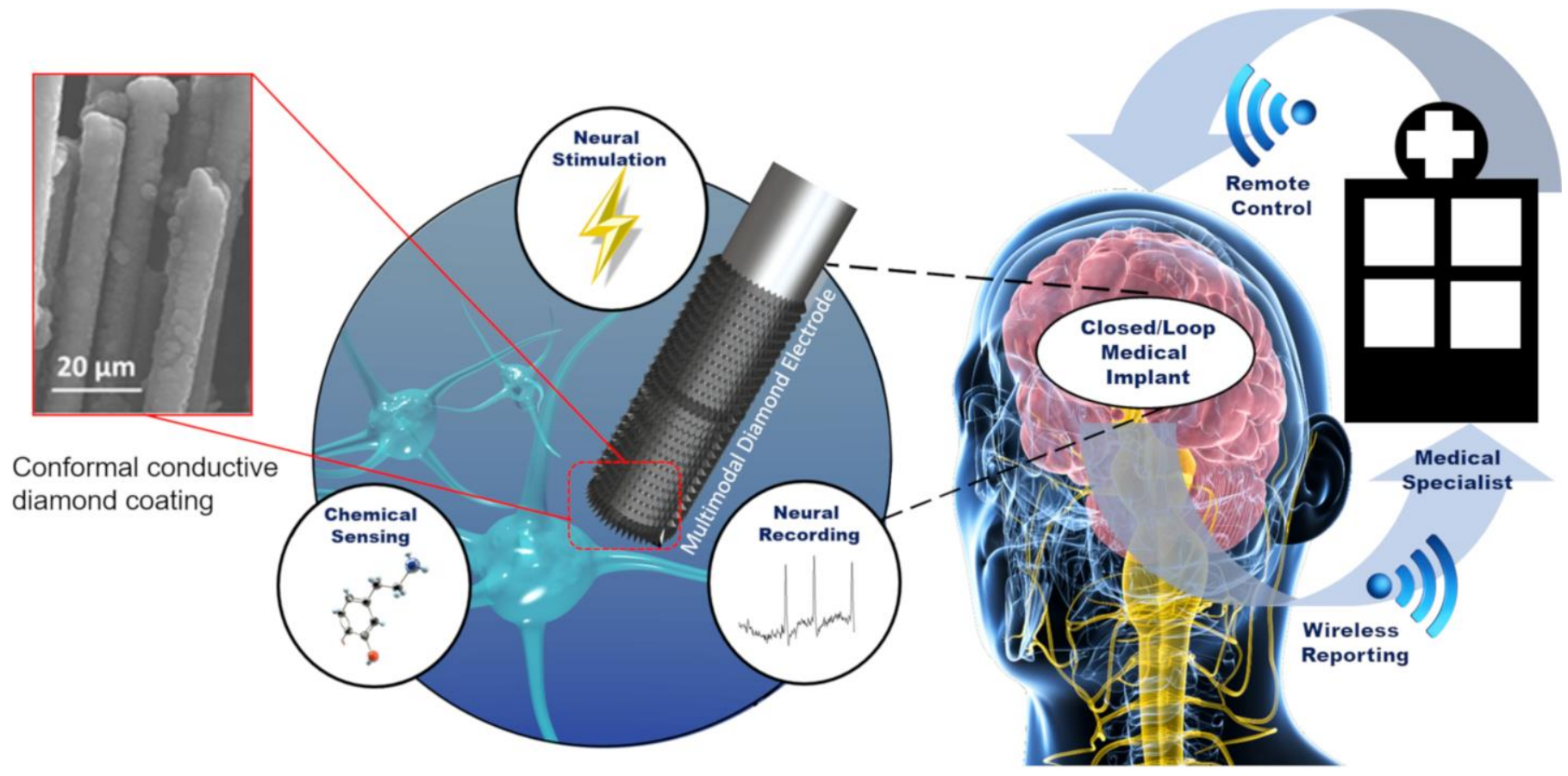

Figure 1. Concept illustration of the capabilities of a fully-featured closed-loop electric medicine device. The implant provides a treatment, monitors its own performance and user disease state, and utilizes monitoring for internal modulation and optimization of treatment. A fully-featured device would also transmit data to an external processing center for deeper interpretation to inform the user's healthcare provider.

Therapies that employ neural stimulation hold great promise but implantable devices face challenges in terms of safety and longevity. These considerations are governed by a range of factors including mechanical compliance, chemical biocompatibility, and electrochemical properties. CFs have been under investigation as promising neural interface electrodes because they exhibit biochemical longevity and are well tolerated in vivo due to their small diameter and flexibility, reducing the risk for infection and formation of glial scars in vivo [6-8]. CFs are also well established as excellent electrodes for neural recording [9] and electrochemical sensing $[10,11]$. They do not, however, possess sufficient electrochemical capacitance for neural stimulation within safe voltage limits. They also suffer from a relatively small "safe potential window", the range of safe voltages within which water splitting does not occur. Electrodes that deviate beyond the water splitting water-window 
during stimulation can cause damage to neural tissue and are considered unsafe. Furthermore, in the case of $\mathrm{CFs}$, etching of the electrode can occur at voltages higher than $+1 \mathrm{~V}$ resulting in rapid degradation of the electrode.

The stimulation properties of CF electrodes can be improved by the addition of a high capacitance coating, leading to lower interfacial impedance and increased safe charge injection capacity (CIC) [12]. Platinum black or conductive polymers such as PEDOT:PSS have been used as the coating layer for improving the electrochemical properties of $\mathrm{CF}$ electrodes but polymer coatings often exhibit degraded performance after repeated stimulation either due to degradation of the coating material or lack of adhesion to the CF surface [9, 13-16].

Previously, we demonstrated that nitrogen-included ultrananocrystalline diamond (NUNCD) is an effective neural stimulation material [17]. This is a significant finding that adds an additional dimension to diamond's recognized exceptional properties in terms of chemical stability, wide water-window, and high resistance to surface fouling. Among all diamond forms, NUNCD is the only form of diamond that has been successfully used for both neural stimulation and recording [18-21]. When an appropriate amount of nitrogen is introduced during chemical vapor deposition (CVD) and post-treated with oxygen plasma [22-24], NUNCD has a CIC as high as $1200 \mu \mathrm{C} / \mathrm{cm}^{2}$ [25]. We have previously demonstrated that this material is well tolerated in vivo [26] and exceptionally stable, even when pushed to the limits of safe operation [17]. In this work, we develop a method to grow this material on single CF microfibers and demonstrate that the resultant electrode can be used safely and effectively for neural stimulation whilst retaining excellent properties for neural recording and electrochemical detection of dopamine. The established stability and robustness of UNCD, even under extreme operating conditions, combined with the small size of CFs makes these electrodes ideal for highresolution, single neuron neural interface electrodes for closed loop medical devices.

\section{Materials and Method}

\subsection{Diamond deposition on Carbon Fibers}

Before nanodiamond seeding, PAN-based CFs (Goodfellow) were electrochemically functionalized with aminophenyl groups on the surface. The electrochemistry was performed in a Teflon cell with a three-electrode set-up using a Gamry Potentiostat (Interface 1000E). An $\mathrm{Ag} / \mathrm{AgCl}$ electrode and a $\mathrm{Pt}$ wire were used as the reference and counter electrode, respectively. A cluster of CFs was connected to a copper wire via silver epoxy. The CFs were first subjected to an acetonitrile solution containing $0.1 \mathrm{M}$ tetrabutylammonium tetrafluoroborate (Sigma) and $1 \mathrm{mM}$ 4-nitrophenyl diazonium tetrafluoroborate (Sigma). Five potential cycles between $0.2 \mathrm{~V}$ and $-0.6 \mathrm{~V}$ vs $\mathrm{Ag} / \mathrm{AgCl}$ was performed at a scan rate of $200 \mathrm{mV} / \mathrm{s}$. The nitrophenyl modified surfaces were sequentially rinsed with acetonitrile, acetone, and deionized water, then exposed to a $0.1 \mathrm{M}$ $\mathrm{H}_{2} \mathrm{SO}_{4}$ solution. The nitrophenyl groups were electrochemically reduced to aminophenyl groups by five potential cycles between $0.5 \mathrm{~V}$ and $-1.5 \mathrm{~V}$ vs $\mathrm{Ag} / \mathrm{AgCl}$ at a scan rate of $200 \mathrm{mV} / \mathrm{s}$. The modified CFs were again rinsed with acetonitrile, acetone and deionized water, and trimmed off from the copper wire to remove silver epoxy for further use.

Modified CFs were seeded with oxygen terminated nanodiamonds via covalent bonding. The preparation of oxygen terminated nanodiamond solution was described elsewhere [27]. Briefly, commercially available nanodiamond powder was first annealed in oxygen at $400{ }^{\circ} \mathrm{C}$ for $5 \mathrm{hr}$ and prepared as a solution with deionized water at a concentration of $1.5 \mathrm{mg} / \mathrm{ml}$. The solution was subsequently ultrasonicated for $1.5 \mathrm{hr}$ and centrifuged for $12 \mathrm{hr}$ to remove large nanodiamond particles and aggregates. According to ZetaSizer measurement, the final nanodiamond showed a concentration of $1.21 \times 10^{8}$ particles $/ \mathrm{ml}$ with zeta potential at $-30 \mathrm{mV}$ and an average particle size of 30-35 nm. The covalent bonding was formed by immersing the treated CFs in nanodiamond solution containing 0.1 M EDC at room temperature overnight. The CFs were then rinsed with deionized water and dried in air. 
After nanodiamond seeding, the CFs were placed in a microwave plasma-assisted CVD system for NUNCD deposition. The fibers were masked with a Molybdenum stage to restrain the growth of diamond on the tips. The deposition was conducted as previously described [23], with a gas mixture of $20 \%$ nitrogen, $79 \%$ argon and $1 \%$ methane, at a temperature of $1000{ }^{\circ} \mathrm{C}$, and deposition time varying between 6 and $22 \mathrm{hr}$.

Finally, single diamond-coated CFs were attached to copper wires by silver epoxy, encapsulated in glass capillaries and pulled to form microelectrodes with a micropipette puller (Model P-2000, Sutter Instrument Co). The length of the fiber exposed was adjusted to $100 \pm 10 \mu \mathrm{m}$ under an optical microscope. The diamondcoated electrodes were then electrochemically oxidized three times by performing cyclic voltammetry in PBS from 0 to $2 \mathrm{~V}$ at a scan rate of $100 \mathrm{mV} / \mathrm{s}$.

\subsection{Surface Characterization}

The nanodiamond solution was characterized with a ZetaSizer (Dynamic Light Scattering technique using a Zetasizer Nano ZS from Malvern). The morphology of the fibers before and after diamond deposition was obtained with SEM/FIB Nova 200 Nanolab, FEG Dual Beam (HV 5 KV, Current 6.3 nA, mode SE). K-edge NEXAFS measurements were conducted at the Soft X-Ray beamline of the Australian Synchrotron. All measurements were conducted in the total electron yield mode, recorded using sample drain current to collect a signal of all secondary electrons and thus data from the bulk of the samples. Double normalization was conducted with an 'IO' gold grid partially inserted into the beam, to monitor fluctuations in beam intensity for all scans, and normalized to a reference photodiode scan for beamline transmission function (primarily for removal of beamline carbon-based absorption characteristics) [28]. Raman spectra were performed on fibers using Renishaw RM 1000 Raman Stellar Pro 514 Modulaser (514 nm excitation source) with $100 \mathrm{~mW}$ power at room temperature. XPS (Thermo- Fisher K-Alpha) was used to obtain the chemical composition of the diamond fibers using an $\mathrm{Al} \mathrm{K} \alpha$ radiation source at a power of $300 \mathrm{~W}$ and a spot size of $400 \mu \mathrm{m}$. High-resolution scans were performed using the flood gun function to compensate for charging at a step size of $0.1 \mathrm{eV}$ and pass energy $50 \mathrm{eV}$. For electrochemical characterization, (CV) and electrical impedance spectroscopy (EIS) measurements were performed using a potentiostat (Gamry, Interface 1000E). All voltammograms were obtained with the electrodes immersed within a Teflon electrochemical cell of three electrodes, where platinum and $\mathrm{Ag} / \mathrm{AgCl}$ are counter and reference electrodes, respectively. All experiments were conducted in $1 \mathrm{M}$ PBS $(0.13 \mathrm{M} \mathrm{NaCl})$ at room temperature without nitrogen gas bubbling. EIS was performed for estimating the specific capacitance of the electrodes. The recording was then fit with a Reap2Cpe circuit [29], as shown in Figure 3S.

\subsection{In Vitro Retinal Ganglion Cell Stimulation}

Calcium imaging data came from Long-Evans rats older than 3 months, of either gender. All experimental procedures conformed to the policies of The National Health and Medical Research Council of Australia (NHMRC) and were approved by the Animal Experimental Ethics Committee of the University of Melbourne (Ethics Approval \#: 1814462). Animals were initially anesthetized with a mixture of ketamine and xylazine prior to enucleation. After enucleation, the rats were sacrificed with an overdose of Letharbarb (intracardiac). To load retinal ganglion cells with calcium indicators, $0.5 \mu 1$ of $20 \mathrm{mM}$ OGB-1 (hexapossium salt dissolved in DI water) was injected into each eye through the optic nerve. The cornea and lens were removed, and the retina was left inside a dish with carbogenated Ames medium overnight at room temperature. The retinas were then cut into three pieces, mounted onto a glass slide with ganglion cell layer up, and held in place with a perfusion chamber and stainless-steel harp fitted with Lycra threads (Warner Instruments). Once mounted in the chamber, the retina was perfused (4-6 ml/min) with heated carbogenated Ames medium $\left(33-35^{\circ} \mathrm{C}\right)$. D-CF electrodes were then placed $200 \mu \mathrm{m}$ above the inner limiting layer. All procedures were conducted under dim light conditions. 
Calcium transients of retinal ganglion cells in response to electrical stimulation were then recorded with an upright confocal microscope (Olympus, FV 1000). The images were obtained with an excitation laser at 473nm through a Nikon Plan Apo 0.75-numerical aperture (NA) x20 objective. Stimuli were delivered using a Ripple system and images were acquired at $7 \mathrm{~Hz}$ and synchronized to the onset of each stimulus. Each stimulus was composed of ten groups of biphasic pulse trains $(60 \mathrm{~Hz}, 0.5 \mathrm{~ms}$ phase duration, cathodic first), with each train composed of ten pulses. Stimuli were delivered ten times at $2 \mathrm{~s}$ intervals and repeated over ten monotonically increasing amplitudes. The percentage of electrically evoked calcium transients was then fit with a sigmoidal curve and the threshold was defined as the stimulation amplitude that yielded a 50\% response. Data processing was performed in Fiji (ImageJ-win64) and Matlab R2014a.

\subsection{In Vitro Retina Ganglion Cell Recording}

The recording capabilities of diamond-coated electrodes were also tested in vitro with a retinal whole mount preparation, similar to that stated in the stimulation session above. No calcium indicator was injected, and the retina was mounted with retinal ganglion cell side up immediately after dissection. Extracellular recordings were obtained by placing the electrode tip in contact with the retinal surfaces. Signals were acquired and amplified (Tucker Davis Technologies: RZ2 base station and PZ2 multichannel recorder), filtered online with band-pass frequencies between 5 and $15 \mathrm{kHz}$, and digitized at $50 \mathrm{kHz}$ for offline analysis. Light responses were obtained using a $\sim 100 \mu \mathrm{m}$ light spots centered at the electrode. The ratio of spikes, one second prior and one second after light stimulation, were compared. Additionally, the instantaneous changes in responses from $1 \mathrm{~s}$ prior compared to $1 \mathrm{~s}$ after a change in illumination were also analyzed. Spikes were detected when the recorded signal crossed a threshold of eight standard deviations computed from the entire recording. A spike cluster analysis [30] was used to classify and group spike waveforms with similar shapes. Once clustered, the SNR was calculated to evaluate the quality of the recordings. The signal quality was defined according to the SNR, which was calculated as the peak-to-peak amplitude of the mean waveform of the cluster divided by twice the standard deviation of the noise [31]:

$\mathrm{SNR}=\left(\mathrm{S}_{-\mathrm{pp}}\right) / 2 \sigma$ Noise

where $\mathrm{S}_{\text {-pp }}$ is the mean peak-to-peak amplitude of the spike amplitude, and $\sigma$ Noise is the standard deviation after all the identified spike waveforms had been removed.

\subsection{In Vivo Visual Cortex Recording}

\subsubsection{Surgery and preparation}

In vivo experiments involved recording using diamond coated electrodes from the primary visual cortex of a Tammar Wallaby (Macropus Eugenii), which is a species that has had its cortex studied extensively using traditional tungsten-in-glass electrodes [32-34]. The electrode testing was conducted as part of ongoing comparative neuroscience projects and was approved by the Animal Ethics Team at the University of Melbourne (approval number, 1714178.1). Before surgery, the animal was given lincomycin (10 mg/kg) and spectinomycin $(20 \mathrm{mg} / \mathrm{kg})$ intramuscularly and paraffin oil $(10 \mathrm{ml})$ orally to reduce intestinal bloating during prolonged anesthesia. Anaesthesia is induced with intramuscular injection of ketamine $(10 \mathrm{mg} / \mathrm{kg})$, medetomidine $(0.015 \mathrm{mg} / \mathrm{kg})$ and methadone $(0.4 \mathrm{mg} / \mathrm{kg})$. During surgery, the animal was given phytomenadione (Koagulon, $10 \mathrm{mg} / \mathrm{kg}$ ) and tranexamic acid (Vasolamin, $100 \mathrm{mg} / \mathrm{kg}$ ) intramuscularly in case of bleeding.

The animal was intubated then connected to a ventilator and anesthesia machine. Inhalation of gaseous isoflurane (1-2\% during surgery) and halothane (0.5\% during recordings) with a 2:1 mix of $\mathrm{O}_{2}$ and $\mathrm{N}_{2} \mathrm{O}$ maintained anesthesia throughout the surgery and experiment. The animal's health was monitored throughout the entire experiment by checking the electrocardiogram (ECG), electroencephalogram (EEG), respiration 
rate, blood pressure, end-tidal $\mathrm{CO}_{2}$ concentration, and oxygen concentration. The animal's body temperature was monitored and maintained by a feedback-controlled heating blanket. Following surgery, the animal was paralyzed with vecuronium bromide (Vecuronium sun; $0.05 \mathrm{mg} / \mathrm{kg}$ for induction followed by a constant intravenous infusion at $0.1 \mathrm{mg} / \mathrm{kg} / \mathrm{h}$ in Hartmann's solution containing $5 \%$ glucose) to prevent eye movements. The intravenous infusion of fluid was delivered through a tail vein at $2.5 \mathrm{ml} / \mathrm{kg} / \mathrm{h}$. Daily intramuscular injections were given to the animal: Atropine $(0.05 \mathrm{mg} / \mathrm{kg})$ to reduce salivation, dexamethasone $(1.5 \mathrm{mg} / \mathrm{kg})$ to prevent cerebral edema and a broad spectrum antibiotic (Clavulox, $0.5 \mathrm{~mL} / \mathrm{kg}$ ) to control infection.

The head of the wallaby was stabilized with a stereotaxic frame and custom-made ear bars. A craniotomy was conducted from $3 \mathrm{~mm}$ to $20 \mathrm{~mm}$ posterior to bregma and approximately $2 \mathrm{~mm}$ to $14 \mathrm{~mm}$ lateral from the midline to access the primary visual cortex (V1) in the left hemisphere. Then a durotomy was performed to expose the brain. A stainless-steel chamber was affixed to the skull with dental cement to stabilize the cortex during recording and prevent cortical tissues from drying out. To maintain moisture on the brain, $4 \%$ agarose in saline was frequently applied to the surface of the brain. Atropine sulfate eye drops $(1 \%)$ were used to dilate the pupils and prevent accommodation. The corneas were protected with zero-power gaseous permeable contact lenses. At the termination of experiments, animals were euthanized by intravenous injection of an overdose of pentobarbitone sodium (Lethabarb; $150 \mathrm{mg} / \mathrm{kg}$ ).

\subsection{Visual Stimuli}

Visual stimuli were generated with a ViSaGe visual stimulus generator (Cambridge Research Systems, Cambridge, UK) and displayed on a calibrated LED monitor (ASUS VG248 resolution 1920x1080 pixels). The LED monitor was viewed monocularly from $30 \mathrm{~cm}$ distance. A custom MatlabTM program was used to generate all visual stimuli (The Mathworks Inc. Natick, MA, USA). During single-unit recording, drifting sinusoidal gratings were presented to determine the preferred orientation of the recorded neurons.

\subsection{Single-Unit Recording}

Multi-unit activity (MUA) was recorded from the primary visual cortex. Recorded signals were amplified and filtered by the computer running the stimulation and recording software. Offline software from KiloSort was used to sort single units from MUA. After spike-sorting, single units could be readily identified. The responses of these units were then correlated with the stimulus presentation.

\subsection{Simultaneous Detection of DA and UA}

CV studies were carried out to study the electrochemical response for different concentrations of DA and UA on electrochemically oxidized D-CF with potentials scanned between $-0.2 \mathrm{~V}$ and $0.8 \mathrm{~V}$ with respect to an $\mathrm{Ag} / \mathrm{AgCl}$ electrode. Electrochemical oxidization of the electrode was performed in $3 \mathrm{mM}$ of $\mathrm{K}_{4}\left[\mathrm{Fe}(\mathrm{CN})_{6}\right]$ in 1 $\mathrm{M} \mathrm{KCl}$ solution from $0 \mathrm{~V}$ to $2 \mathrm{~V}$ with 100 repetitive cycles and scan rated $1 \mathrm{Vs}^{-1}$. It is noted that the limitation of detection (LOD) is determined using the following formula: $\mathrm{LOD}=$ mean blank $+3 \sigma$ blank.

\section{Results and discussion}

\subsection{Diamond Deposition on Carbon Fiber}

Diamond coatings on CF have been attempted by many researchers before but with limited success [35]. The difficulty is mainly associated with the etching of CF under the CVD environment necessary for diamond deposition [36]. In order to protect the CF during CVD, we developed a new method for pre-seeding CF with covalently bound nanodiamonds before diamond growth (Figure 2, a and b). Covalent bonding of nanodiamonds was achieved by tethering nanodiamonds to the CF surface via a grafted aryl amine linker layer subsequently coupled to carboxylic acid terminated nanodiamonds via an amide bond (Figure 2c) and described in more detail in the supplementary methods section. The method is derived from the previous work 
of Garrett et al. who used similar methods to couple carbon nanotubes to graphitic substrates [37]. To our knowledge, the method has not previously been applied to nanodiamonds. Successful covalent attachment of the aryldiazonium layer was confirmed by observation of the cyclic voltammogram (CV) during electrochemical deposition and subsequent reduction of the nitro group (Figure 1S a-b). X-Ray Photoelectron Spectroscopy (XPS) analysis confirmed both the presence of an aminophenyl film on the CF layer and the presence of amine groups following covalent coupling of carboxylic acid functionalized nanodiamonds (Figure 1S c-e).

(a)

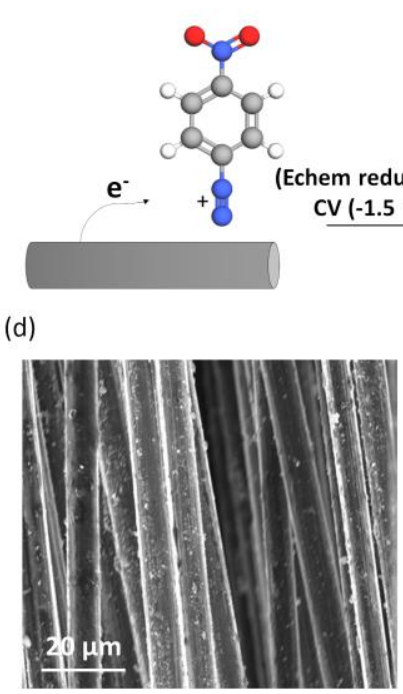

(g)

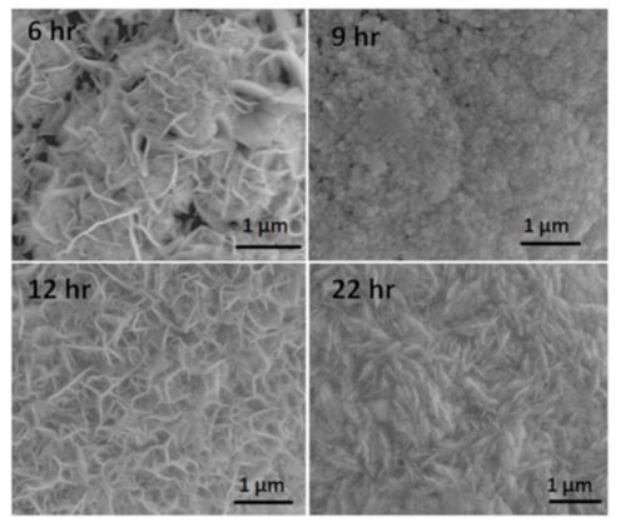

(e) (c)
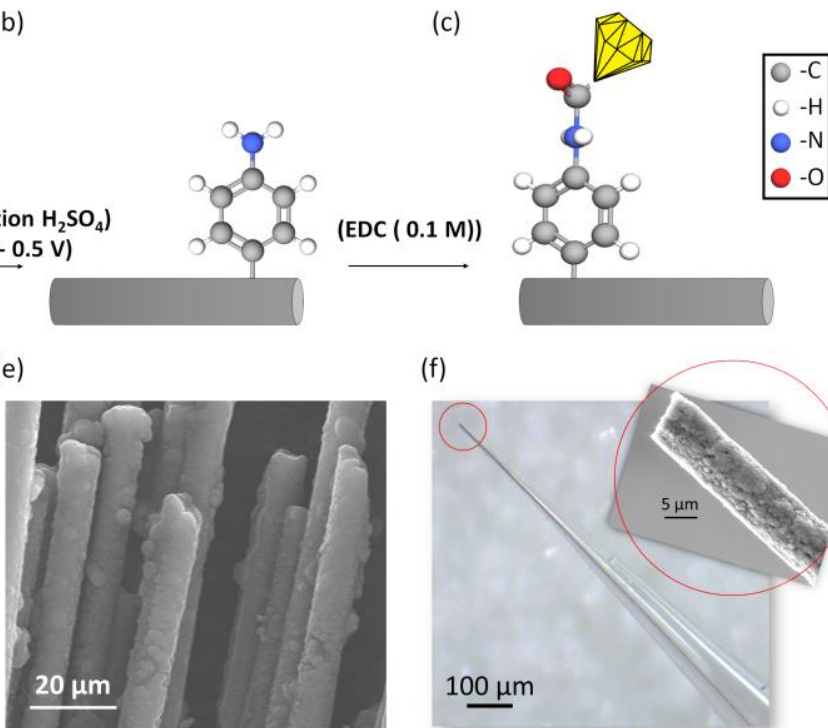

(f)

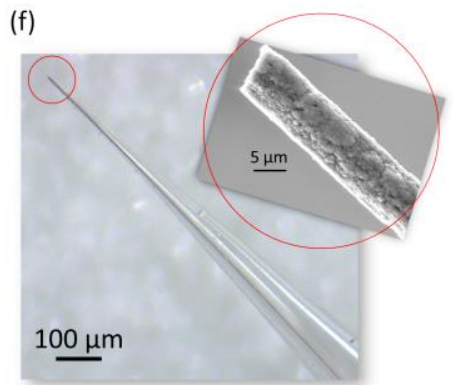

(h)

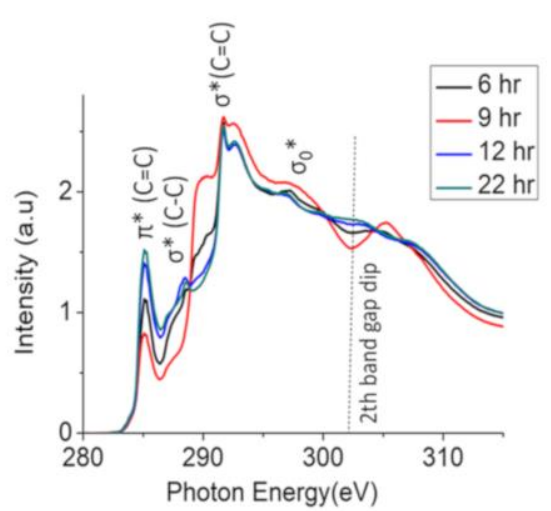

(i)

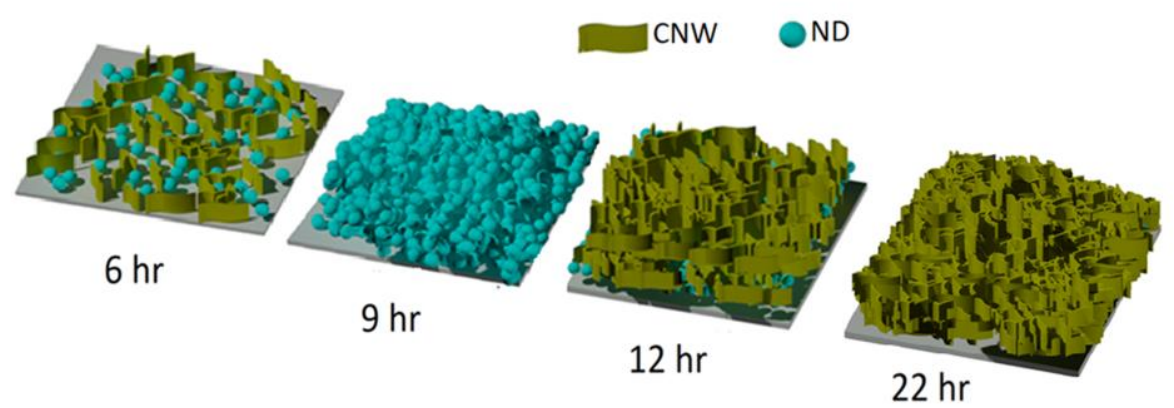

Figure 2. NUNCD deposition on CF. (a) Electrochemical grafting of nitrophenyl diazonium to CF and (b) subsequent electrochemical reduction to an aminophenyl linker layer. (c) Covalent coupling of carboxylic acid 
functionalized nanodiamonds to form a CVD seed layer. SEM images of (d) seeded and (e) diamond-coated CFs. (f) Optical image of a single diamond-coated CF electrode encapsulated in a glass pipette with the SEM image of the electrode tip shown in (f) inset. (g) High magnification SEM images of NUNCD surface after deposition times between 6 and 22 hrs. The surface is composed of a mixture of carbon nanowalls and ultrananocrystalline diamond. NEXAFS spectrum from each surface is shown in (h). The schematic change of surface structure is summarized in (i).

NUNCD growth was performed on the covalently bonded surfaces for various growth times. With the sample grown for $6 \mathrm{hr}$, carbon nanowalls (CNW) featured strongly in the resultant films. High-resolution SEM images (Figure $2 \mathrm{~g}$ ) reveal that the surface is covered by ribbon structured $\mathrm{CNW}$ aligned perpendicular to the $\mathrm{CF}$ surfaces, with ultrananocrystaline diamond (UNCD) structures seen between the ribbons. It is reported that the presence of hydrogen at the diamond surface may play an important role in the CNW phase nucleating directly on the diamond surface [38]. After $9 \mathrm{hr}$, NUNCD appears to have covered the surface of the CNWs. During this stage, the hydrogen atoms from the gas mixture in the CVD may react with $\mathrm{sp}$ or $\mathrm{sp}^{2}$ carbon sites on the surface of the CNW and convert them into $\mathrm{sp}^{3}$ bonded carbon (diamond) [38]. At $12 \mathrm{hr}$, we observed re-nucleation and regrowth of CNWs on the NUNCD surface. Our results indicate that after $12 \mathrm{hr}$, the condition is more suitable for nucleating and growing CNWs rather than nanodiamond and the nanosheet grows much faster in the direction parallel to the sheet due to the very high surface mobility of incoming $\mathrm{C}$ atoms or $\mathrm{CH}_{\mathrm{x}}$ radicals, and polarization of the graphitic layers [39]. After $22 \mathrm{hr}$ deposition, a large number of CNW rendered a needle-like morphology (Figure 2g).

NEXAFS (Figure $2 \mathrm{~h}$ ) is sensitive to the bonding structure $\mathrm{sp}^{2} / \mathrm{sp}^{3}$ and thus determines the type of carbon in a given area [40]. The peak located around $285 \mathrm{eV}$ corresponds to the transition $\mathrm{C} 1 \mathrm{~s} \rightarrow \pi^{*}$ for the $\mathrm{sp}^{2} \mathrm{C}=\mathrm{C}$ bond $[41,42]$. At the surface of pure diamond due to surface reconstruction [43]. The $\mathrm{sp}^{2}$ peak intensity increased from the lowest to the highest attributed to the CF-NUNCD for 9hr, $6 \mathrm{hr}, 12 \mathrm{hr}$, and $22 \mathrm{hr}$, respectively. The peak located around $289 \mathrm{eV}$ is associated with core-level excitons in diamond material $[41,44]$ and the start of the conduction band edge. For pure diamond, the $\sigma^{*}(\mathrm{C}=\mathrm{C})$ resonance should dominate, and the ratio of the intensities gives a figure of merit for the level from $\mathrm{sp}^{2}$ to $\mathrm{sp}^{3}$ bonding [40].

The edge feature at $292 \mathrm{eV}$ is associated with a $\sigma^{*}(\mathrm{C}=\mathrm{C})$ resonance, indicating graphitic materials. Conversely, an edge at $289 \mathrm{eV}$ relates to $\sigma^{*}$ transitions at the onset of the conduction band edge. The remainder of the $\sigma^{*}$ region above $289 \mathrm{eV}$ can be difficult to interpret, except for the presence of a clear second absolute band gap dip at $302 \mathrm{eV}$ [42] which is unequivocally from the diamond. Thus, it can be seen that the $9 \mathrm{hr}$ sample shows a clear mixture of crystalline diamond and graphitic bonding. The $6 \mathrm{hr}$ sample shows a lower proportion of diamond-related features at $289 \mathrm{eV}$ and $302 \mathrm{eV}$, while the other two samples show no clear evidence of diamond-like character. The relative intensity of the $\pi^{*}$ feature for all four samples scales inversely with the diamond-associated $\sigma^{*}$ features, confirming this analysis. Raman spectra and XPS analysis also suggest these changes during the growth process (Figure $2 \mathrm{~S}$ and Table $1 \mathrm{~S}$ ).

\subsection{Improved Charge Injection Capacity for Neural Stimulation}

To be used for neural stimulation, single fiber electrodes were fabricated by first attaching the fibers onto a copper wire via silver epoxy and insulated inside a pulled glass capillary such that a $100 \pm 10 \mu \mathrm{m}$ length of fiber was exposed to the solution.

CIC is a figure of merit used in neural stimulation research to describe the maximum amount of charge that can be injected during a single stimulation pulse before unsafe voltages occur on the electrode (i.e. before the 
water-window is exceeded). In this work, CIC was calculated using capacitance and water-window according to the following formula (Equation 1):

$$
C I C=\frac{C_{d l} \times V_{m}}{G S A}
$$

where $\mathrm{C}_{\mathrm{dl}}$ is specific electrochemical capacitance, $\mathrm{V}_{\mathrm{m}}$ is the voltage threshold to electrolysis of water and GSA is the geometric surface area of the electrode exposed to the solution. Here $\mathrm{V}_{\mathrm{m}}$ was estimated from cyclic voltammograms in PBS (Figure 3a) and set at $0.4 \mathrm{~V}$ for $\mathrm{CF}$ and $1.2 \mathrm{~V}$ for all diamond-coated samples, the same as previously reported [23]. $\mathrm{C}_{\mathrm{dl}}$ was measured by electrochemical impedance spectroscopy (EIS), fitted to an equivalent circuit model as shown in Figure 3S. The $\mathrm{CIC}$ for diamond-coated $\mathrm{CF}$ after different deposition times were measured to determine the optimal surface for neural stimulation. The GSA of the exposed fibers was estimated using SEM imaging, modeling the electrode as a smooth cylinder, and not taking surface roughness into account.

Among electrodes, the specific capacitance was largest on coated CF with 9hr NUNCD deposition (D-CF), when most of the surface was covered by $\mathrm{sp}^{3}$ bonded carbon (see Section 2.1 and Table S2). The specific capacitance increased approximately eighty fold from $0.263 \pm 0.168 \mathrm{mF} / \mathrm{cm}^{2}$ on CF to $20.90 \pm 10.30 \mathrm{mF} / \mathrm{cm}^{2}$ on D-CF ( $\mathrm{P}<0.05$, unpaired student t-test, Figure $3 \mathrm{c}$ ). As $\mathrm{CF}$ has significantly smaller water-window than diamond-coated samples, CIC of D-CF was 238 times larger than that of $\mathrm{CF}$, increasing from $0.105 \pm 0.067$ $\mathrm{mC} / \mathrm{cm}^{2}$ to $25.08 \pm 12.37 \mathrm{mC} / \mathrm{cm}^{2}$ (Figure $3 \mathrm{~d}$ ). Figure $3 \mathrm{~b}$ shows light microscope images of D-CF and CF electrodes during stimulation and illustrates the difference between the CIC for the two materials. For bare $\mathrm{CF}$, water splitting, and gas bubble formation occurred at $150 \mu \mathrm{A}$ during a $0.5 \mathrm{~ms}$ square wave current pulse. In contrast, the D-CF electrodes exhibit no bubble formation at $400 \mu \mathrm{A}$ pulse amplitude (Figure $3 \mathrm{~b}$ ). A range of other commonly used neural stimulation electrode materials is compared with D-CF in Table 1.

Table 1. A range of other commonly used neural stimulation electrode materials compared with D-CF.

\begin{tabular}{|c|c|c|c|c|}
\hline Types of material & $\begin{array}{c}\text { Double layer } \\
\text { Capacitance } \\
\left(\mathrm{mF} / \mathrm{cm}^{2}\right)\end{array}$ & $\begin{array}{c}\text { Charge } \\
\text { Capacitance } \\
\left(\mathbf{m C l} / \mathrm{cm}^{2}\right)\end{array}$ & $\begin{array}{c}\text { Water- } \\
\text { Window (V) }\end{array}$ & Reference \\
\hline N_UNCD & $0.038 \pm 0.005$ & $0.1-0.2$ & $-1.1-1.1$ & [23] \\
\hline Reduced graphene oxide fiber & $0.016 \pm 0.001$ & $14 \pm 0.9$ & $-1-0.9$ & [45] \\
\hline oxygen plasma activated NUNCD & 1 & 1 & $-1.1-1.1$ & [25] \\
\hline Boron doped polycrystalline diamond & 0.087 & --- & $-0.8-1.4$ & [46] \\
\hline Titanium nitride (TiN) & --- & 4.45 & $-1.4-1.6$ & [47] \\
\hline Platinum & --- & $0.15-5.57$ & $-0.8-0.8$ & {$[48]$} \\
\hline D-CF & $20.90 \pm 10.30$ & $25.08 \pm 12.37$ & $-1.8-1.2$ & This work \\
\hline
\end{tabular}

\subsection{Neural Stimulation with D-CF.}

The capability of these electrodes to stimulate neurons was tested using live, wholemount explanted rat retina. A calcium indicator, (Oregon Green 488 BAPTA-1), was loaded into Long-Evans rat retina via optic nerve injection and overnight diffusion. The retinas were kept perfused and alive during loading and during the 
subsequent experiment. The successful loading of calcium indicator cells was confirmed via confocal microscopy, as shown in Figure 3e. The image shows effective staining of retinal ganglion cell somas, axon bundles, and blood vessels. Test electrodes were then placed $200 \mu \mathrm{m}$ above the inner limiting membrane (top surface of retina), which mimics the condition when retinal prosthetics are implanted in patients when there is a gap between the electrodes and retinal surface due to limitations of the surgical implantation technique.

(a)

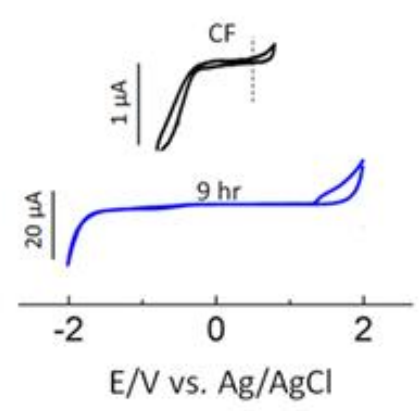

(b) $\mathrm{CF}$

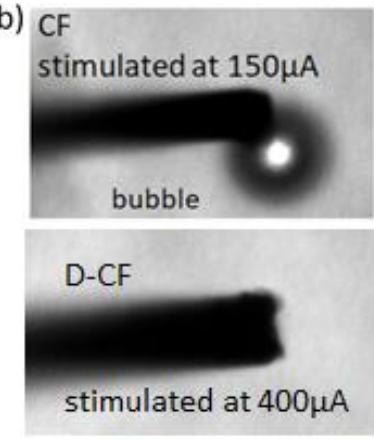

(e)

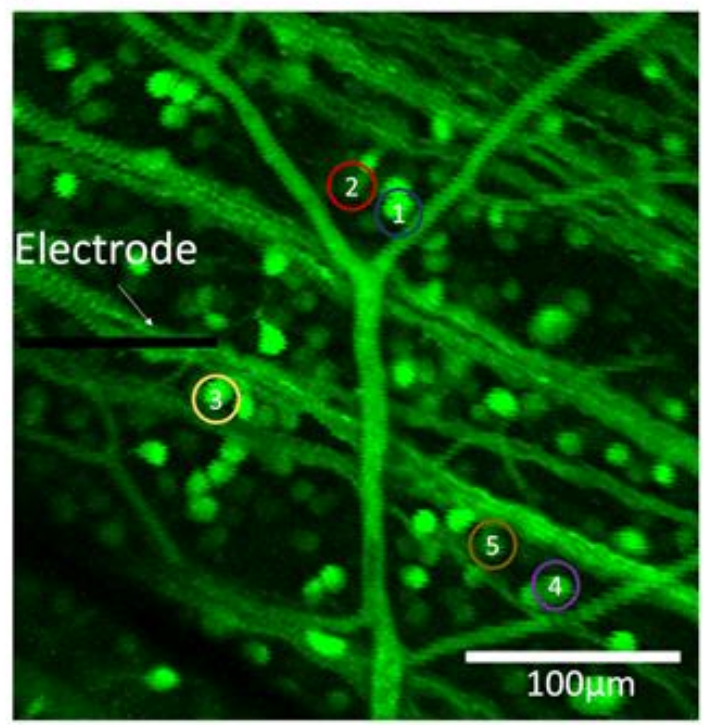

(c)
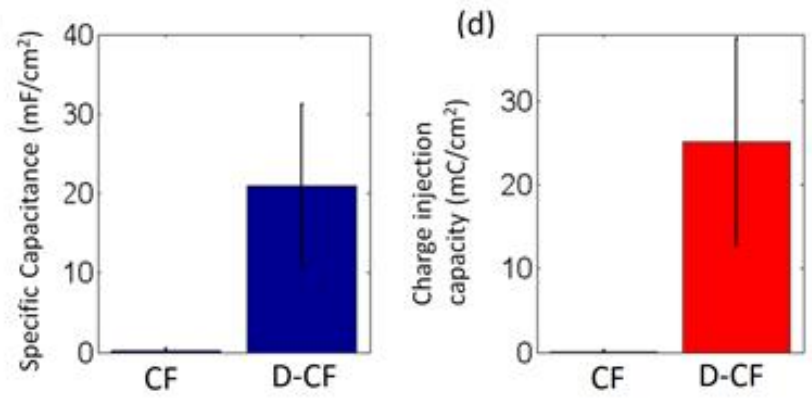

(f)

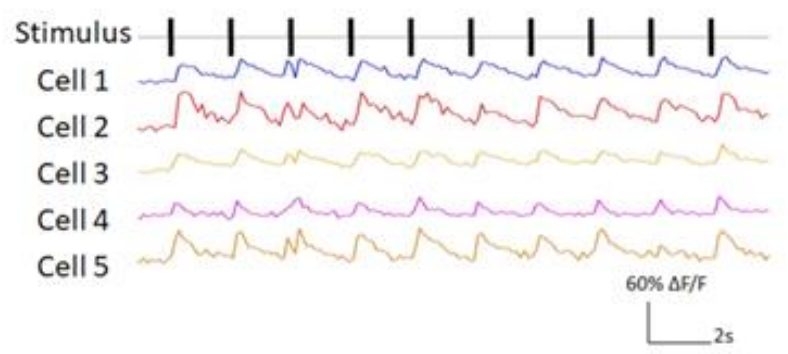

(g)

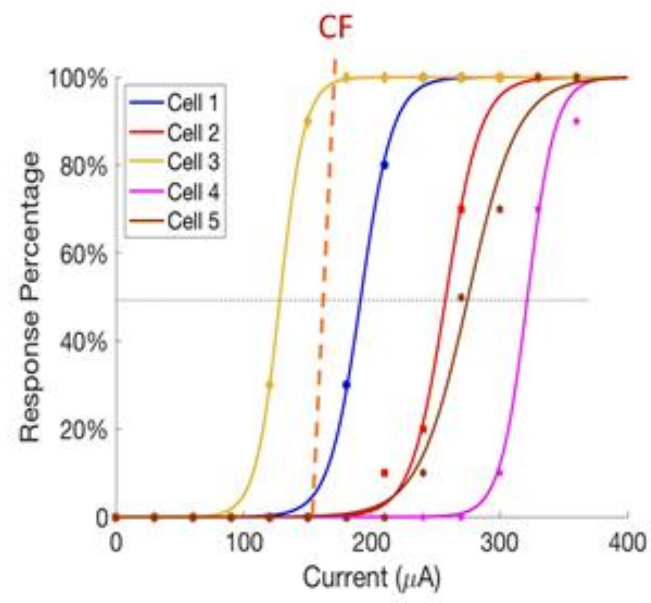

Figure 3. Improved CIC on D-CF electrodes for neural stimulation. (a) Cyclic voltammograms used for estimating the water-window for $\mathrm{CF}(0.4 \mathrm{~V})$ and D-CF electrode $(1.2 \mathrm{~V})$. (b) Microscope images of CF and D-CF. The images show CF electrolyzing water and forming gas bubbles with $150 \mu \mathrm{A}$ amplitude pulses compared to D-CF electrodes where no bubbles form even with $400 \mu \mathrm{A}$ pulses. Pulse duration: $0.5 \mathrm{~ms}$. (c) Specific capacitance increased from $0.263 \pm 0.168 \mathrm{mF} / \mathrm{cm}^{2}$ on $\mathrm{CF}$ to $20.90 \pm 10.30 \mathrm{mF} / \mathrm{cm}^{2}$ on D-CF electrodes. (d) CIC of D-CF was 238 times larger than that of CF, which increased from $0.105 \pm 0.067 \mathrm{mC} / \mathrm{cm}^{2}$ to $25.08 \pm 12.37 \mathrm{mC} / \mathrm{cm}^{2}$. D-CF electrodes were then used for stimulation of retinal ganglion cells. (e) Fluorescence images were collected in real time during electrical stimulation. The D-CF electrode successfully activated retinal ganglion cells, as indicated in (f) and (g). (f) Calcium transients of the example ganglion cells circled in (e). (g) A sigmoidal curve was fitted to the response rate for each neuron. The neurons current threshold was defined as the current required to elicit a 50\% response. The dashed red line indicates that current amplitude where water splitting occurred for CF. 
Figure $3 f$ shows recorded calcium transients from the five example cell somas indicated in Figure 5e during a stimulus train, composed of ten groups of pulses, each group starting at times indicated by the black bars in the line labeled "Stimulus". Each group of pulses is made up of ten cathodic first biphasic pulses with the same current amplitudes, $0.5 \mathrm{~ms}$ phase duration and $0.05 \mathrm{~ms}$ interphase gap at a frequency of $60 \mathrm{~Hz}$. An effective stimulus causes an influx of calcium indicator into the soma and hence an increase in fluorescent intensity. Electrically evoked responses were detected by high pass filtering the fluorescence intensity of each RGC. As previously described [49] filtering was performed by convolving the signals with a difference filter, [2 1 -1 -2]. The rapid changes in fluorescence was determined by thresholding at $1.5 \mathrm{x}$ the RMS noise level and then temporally correlating with the stimuli.

Figure $3 \mathrm{~g}$ shows the response curves for the example ganglion cells, which indicate the percentage likelihood of successful stimulation at various stimulation pulse current amplitudes. A stimulation efficacy of $100 \%$ was achieved at $150 \mu \mathrm{A}$ for the most responsive neuron (cell 3) and approximately $380 \mu \mathrm{A}$ for the least responsive neuron (cell 4). Uncoated CFs are not capable of safely delivering more than $150 \mu \mathrm{A}$ (dashed red line) and would therefore only be able to safely stimulate the most sensitive neuron measured.

The threshold for stimulation of each neuron was defined as the current required to achieve $50 \%$ of the maximum (saturated) response and was estimated by fitting a sigmoidal curve to the response percentage of the cells under different stimulation amplitudes. The measurements were recorded using three electrodes on three pieces of retina, yielding an average stimulation threshold of $255.98 \pm 59.38 \mu \mathrm{A}$ ( 51 activated neurons), $261.93 \pm 50.03 \mu \mathrm{A}$ (40 activated neurons) and $292.55 \pm 79.89 \mu \mathrm{A}$ (46 activated neurons), respectively. It is likely that different thresholds would be measured for different pulse durations and conditions. 


\subsection{High-Quality Single-Unit Neural Recording}

(a)

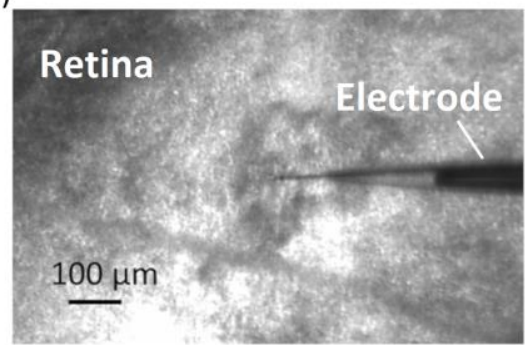

(d) (b)

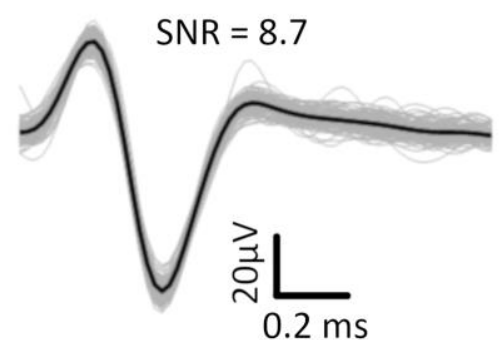

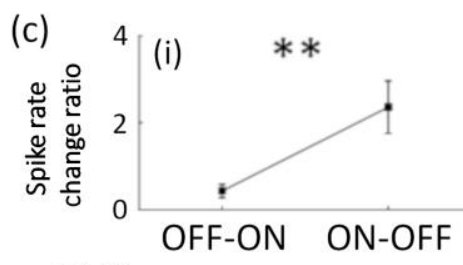

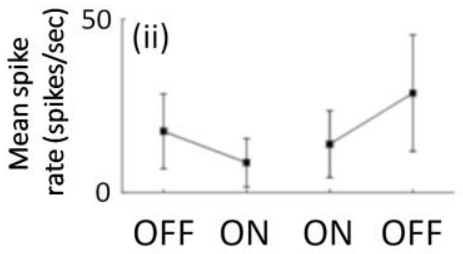

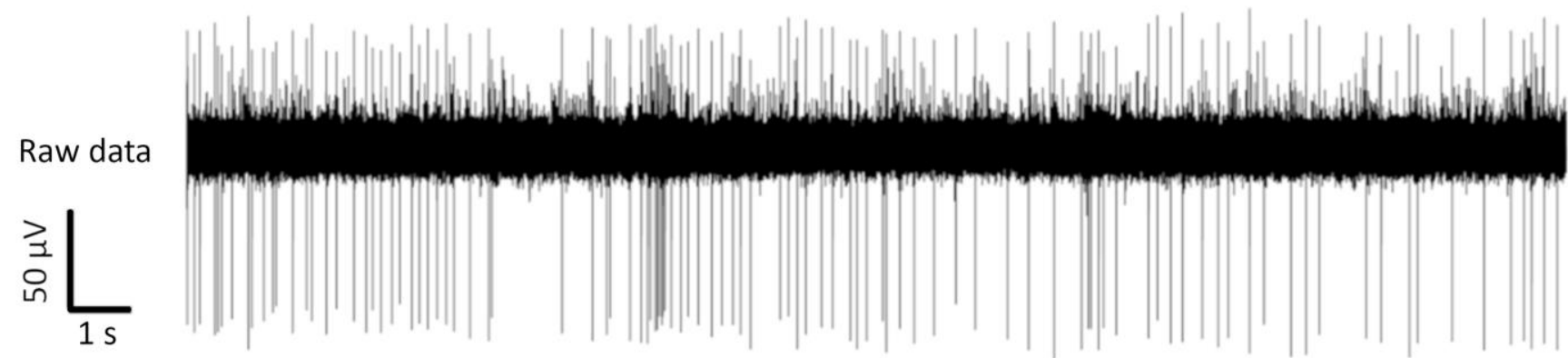

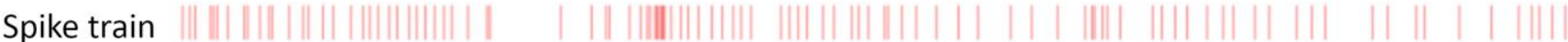
Light stimuli

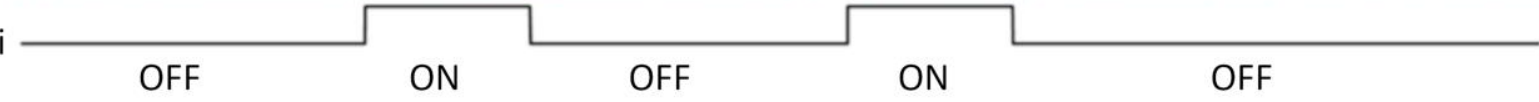

(e)

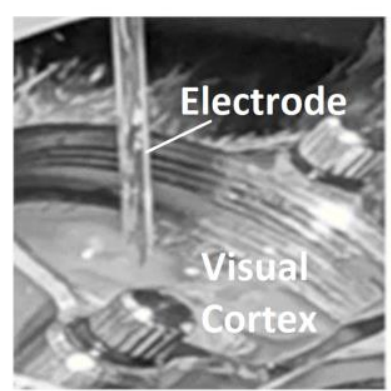

(f)

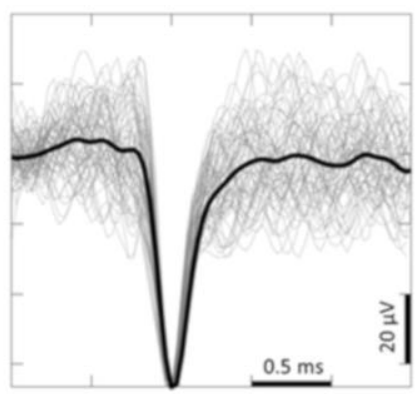

(g)

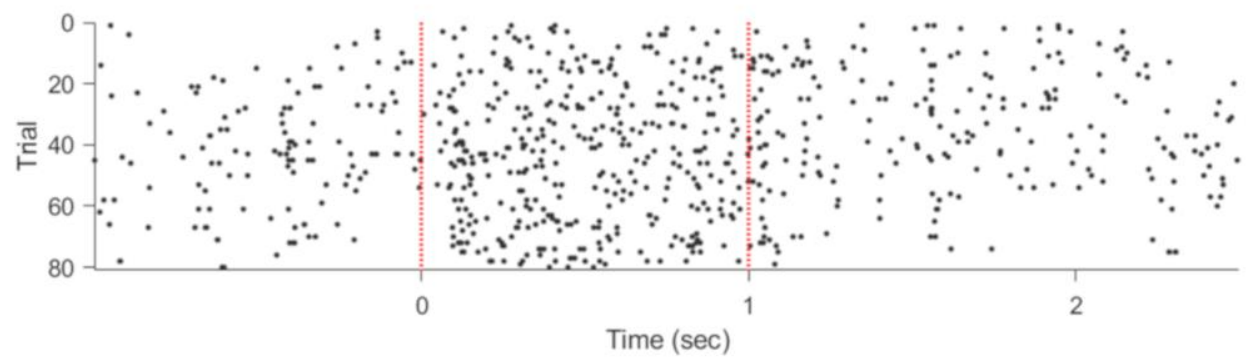

(h)

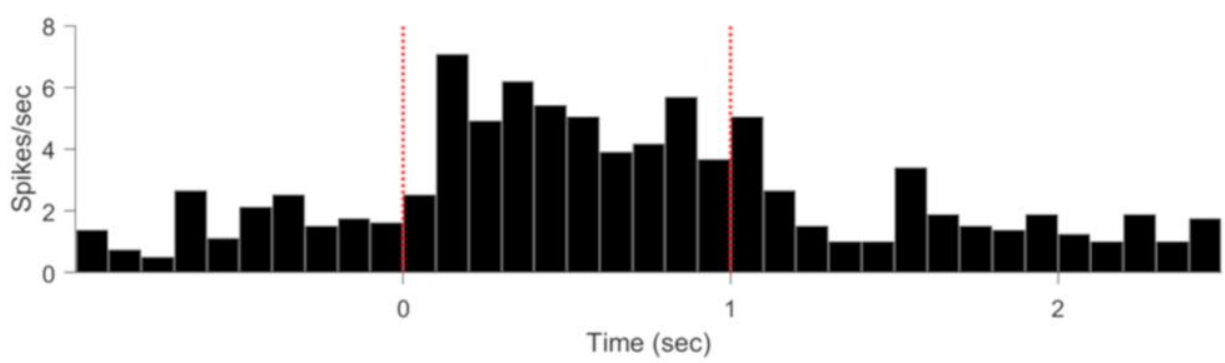


Figure 4. Single-unit recording from an in vitro retinal ganglion cell (a-d) and an in vivo visual cortical neuron (e-h) in response to light stimulation. (a) D-CF electrodes were placed in contact with the surface of the excised retina. (b) Individual spike waveforms from a representative recorded cell (grey traces) and the average spike waveform (black trace) is shown for a single response trial. The average spike amplitude for this trial was $61.76 \pm 1.58 \mu \mathrm{V}$ and had a signal-to-noise ratio (SNR) of 8.7 (c (i)) The change in firing rate of the recorded cell during the transition from light off-to-on and light on-to-off across four trials. A ratio greater than one corresponds to an increase in firing rate and less than one corresponds to a decrease. (c (ii)) The instantaneous firing rate during the transitions from light off-to-on and from off-to-on. Error bar represents standard deviation. ${ }^{*} \mathrm{p}<0.01$ (Student t-test). (d) A representative trial showing the neural response to light stimulation. (e) D-CF electrodes were driven $500 \mu \mathrm{m}$ into the cortex perpendicular to the cortical surface using a piezoelectric drive. (f) Spike waveforms of a single unit recorded from the visual cortex. The grey traces indicate individual spike waveforms and the black trace indicates the mean spike waveform. (g) Raster plot containing the responses of the single unit to eighty-six trials of a $3.5 \mathrm{~s}$ segment of a moving grating pattern. The red dotted lines at zero shows the onset and at $1 \mathrm{~s}$ the offset of the moving grating. (h) Post-stimulus time histogram from the single unit with bins of $0.1 \mathrm{~s}$. Time at zero is stimulus onset (first red dotted line) and stimulus offset $1 \mathrm{~s}$ after (second red dotted line). The $\mathrm{X}$ and $\mathrm{Y}$ axes represent time (s) and spike rate (spikes / s), respectively.

The recording capability of D-CF electrodes was evaluated with explanted rat retinas in vitro (Figure 4a-d), and separately in wallaby visual cortex in vivo (Figure $4 \mathrm{e}-\mathrm{h}$ ). When testing with explanted retinas in vitro, the cell responded with an increase in firing rate when the light was turned off and a decrease in firing rate when the light was turned on (Figure 4b-d). Figure 4a shows the electrode placed into contact with the retinal surface. Figure $4 \mathrm{~b}$ shows the individual spike waveforms for a recorded cell (grey traces) and the average spike waveform (black trace). Spike-sorting was conducted using wave cluster [30] and only one neuron was identified. The mean spike amplitude for this recording was approximately $61.76 \pm 1.58 \mu \mathrm{V}$.

The RMS noise from D-CF electrodes was approximately $2 \mu \mathrm{V}$ in the extracellular solution, significantly lower than equivalent $\mathrm{CF}$ electrodes $(50 \mu \mathrm{V})$ (Figure $4 \mathrm{~S}$ ). We noticed an improvement over time in the recording quality of the electrode. The SNR for the trial shown in Figure $4 \mathrm{~b}$ was 8.7. The mean SNR across three light response trials was $6.36 \pm 2.37$, which is comparable to previously reported values using a $15 \times 15$ $\mu \mathrm{m}$ NUNCD electrode (SNR: 7.25) [50]. Figure 4c shows that the firing frequency of the recorded cell was significantly higher when the light was off compared to on indicating this was an OFF-ganglion cell. The recorded response for one trial and the spike train relative to the light stimulus are shown in Figure $4 \mathrm{~d}$. We repeated the experiment with three electrodes, all of which were able to record neural spikes.

To establish the efficacy of D-CF electrodes for high-resolution cortical recording, an experiment was conducted with the electrodes inserted into the visual cortex of an Australian marsupial (Tammar Wallaby, Macropus Eugenii) as part of ongoing comparative investigations. Wallaby has been shown to have a primary visual cortex (V1) with very similar anatomical and physiological properties to other mammals with highresolution vision, such as primates and cats [32-34]. In accordance with the principles of minimization of the use of experimental animals and to maximize scientific output from a single animal, the experiment was conducted at the conclusion of an approved long-term acute experiment with other experimental aims.

D-CF electrodes were driven $500 \mu \mathrm{m}$ into V1 perpendicular to the cortical surface using a piezoelectric drive (Figure 4e). Three electrodes were used in a single animal and all produced clear action potentials that could be readily extracted from the background noise, using spike-sorting algorithms (Figure 4f). To make sure that the potentials were unequivocally from visual neurons we used moving grating patterns on an LCD display to 
activate the visual neurons in V1. Figure $4 \mathrm{~g}$ shows a raster plot generated by eighty presentations of an optimally oriented grating moving through the receptive field of a V1 neuron. The moving stimulus started at time 0 and finished at time 1. Before and after the stimulus the random firing of the cell's spontaneous spikes can be readily identified. Around $0.1 \mathrm{~s}$ after stimulus onset there was a clear increase in spike rate, which dropped off approximately $0.1 \mathrm{~s}$ after stimulus offset. The timing of the response is clearly revealed by the peristimulus time histogram (Figure 4h). In this recording, SNR (3.8) was comparable to other recording electrodes used in vivo such as parylene C coated platinum electrodes (2.77) [51], PEDOT/CNT (3 \pm 0.6$)$ [31], and the electronic depth control probe $(2.48 \pm 2.68)$ [52].

\subsection{High Sensitivity Simultaneous Dopamine and Uric Acid Detection}

An important functionality of future devices will be the ability to sample chemical environments in vivo. Glucose monitors in closed-loop insulin pumps for people with diabetes are a current example of the benefit of such technologies. Future devices may well monitor local drug concentrations or monitor endogenous biomarkers to evaluate device efficacy or disease progression. Dopamine (DA) is a neurotransmitter that is commonly used to evaluate a material as a neurochemical sensor and is a generic indicator of a material's ability to detect catecholamines, an important class of biomolecules. Figure 5a shows that after electrochemical oxidation of D-CF microelectrodes, the $\mathrm{K}_{3}\left[\mathrm{Fe}(\mathrm{CN})_{6}\right]$ (ferricyanide, a generic in vitro redox test molecule) oxidation peak shifts to a higher peak voltage. This indicates an increase in carboxyl group concentration on the electrode surface. It has also been shown that oxygen-containing functional groups facilitate $\mathrm{Fe}^{2+} /^{3+}$ electron transfer in ferri/ferrocyanide [53]. Oxidized diamond electrodes are also appropriate electrodes for biosensing, especially DA, an important neurotransmitter critical to our attention, learning and memory. The electrode also should be capable of accurately detecting DA under the interference of Uric acid (UA), often oxidized at potentials close to that of DA, hence it acts as an interferant [52]. 
(a)

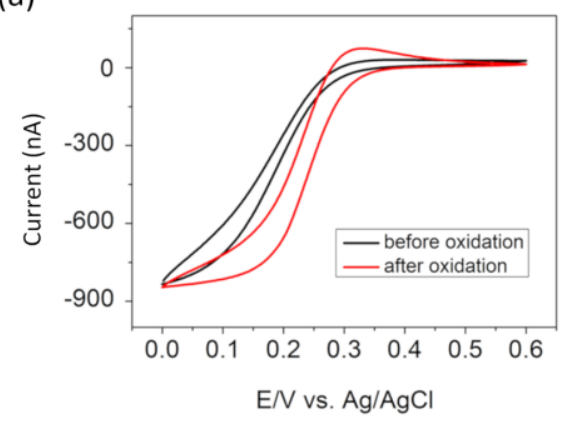

(c)

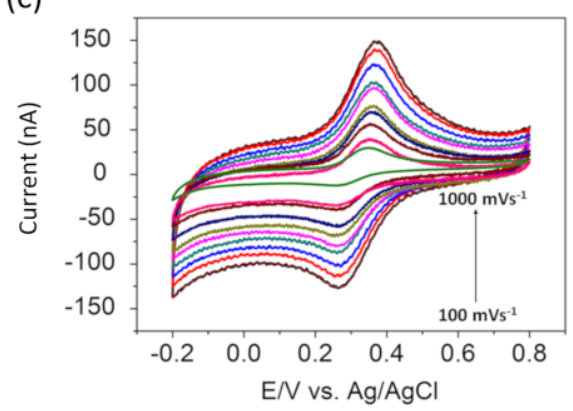

(e)

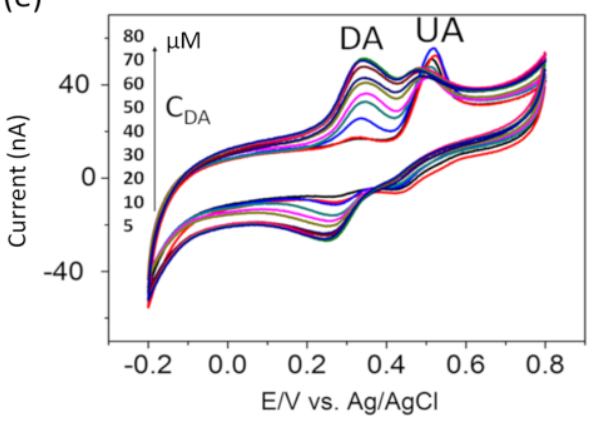

(g)

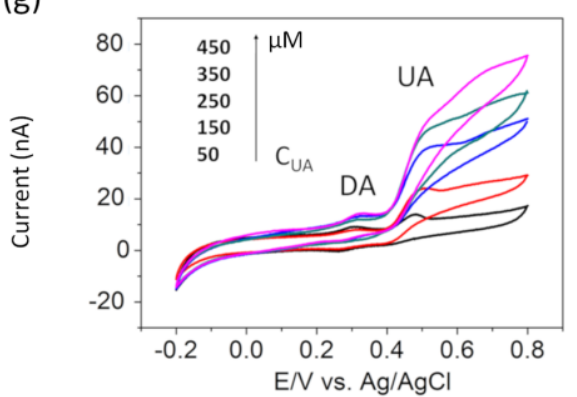

(b)

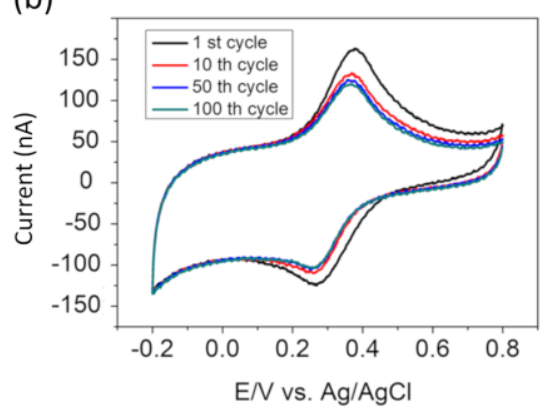

(d)

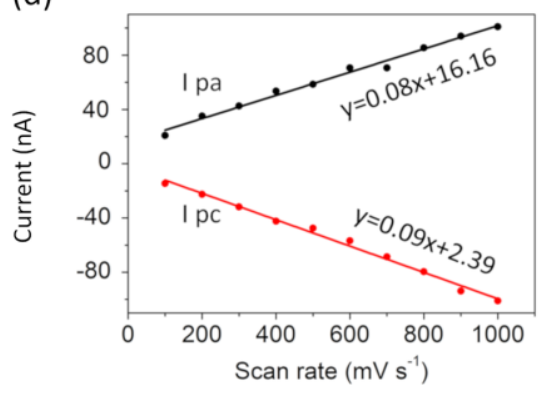

(f)

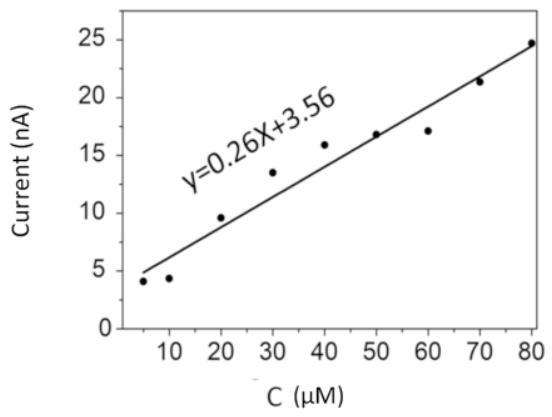

(h)

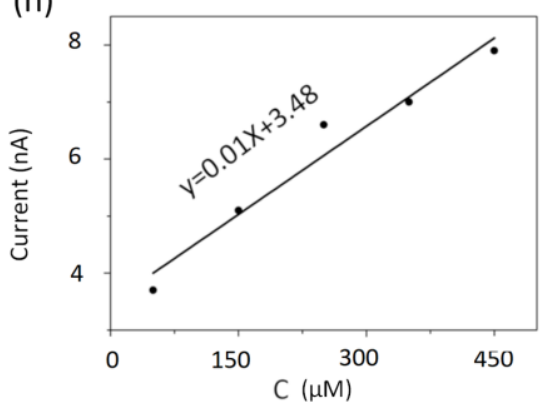

Figure 5. Simultaneous detection of dopamine and Uric acid using cyclic voltammetry (a) Cyclic voltammograms of $3 \mathrm{mM} \mathrm{K}{ }_{3}\left[\mathrm{Fe}(\mathrm{CN})_{6}\right]$ in $1 \mathrm{M} \mathrm{KCl}$ using D-CF before and after electrochemical oxidization of the surface, scan rate $100 \mathrm{mVs}^{-1}$. (b) Repetitive cyclic voltammetry response for $0.1 \mathrm{mM} \mathrm{DA}$ in $1 \times \mathrm{PBS}$ for 100 cycles, scan rate $1 \mathrm{Vs}^{-1}$. (c) Cyclic voltammetric response on D-CF in $0.1 \mathrm{mM}$ DA in a PBS at (c) different scan rates and (d) their corresponding oxidation and reduction peak calibration plots. (e) Cyclic voltammetric curves of $100 \mu \mathrm{M}$ UA in the presence of different concentrations of DA; (f) with their corresponding oxidation peak calibration plot, (g) cyclic voltammetric curves of $10 \mu \mathrm{M}$ DA in the presence of different concentrations of UA, (h) with corresponding calibration curves (scan rate $100 \mathrm{mVs}^{-1}$ ). 
To infer the reproducibility of the sensor, $0.1 \mathrm{mM}$ of DA was added to the electrolyte solution, and the oxidative peak current was noted during 100 cycles at a scan rate of $100 \mathrm{mVs}^{-1}$. As shown in Figure $5 \mathrm{~b}$ the peak current showed a $25 \%$ decrease after $10 \mathrm{CVs}$ but remained stable for subsequent $90 \mathrm{CVs}$. The kinetics of the electrochemical reactions were studied by examining the influence of scan rate on the CV redox peak current and potential of $0.1 \mathrm{mM}$ DA in PBS using different voltage scan rates (Figure 5c). A linear fit of peak current against scan rate (Figure 5d) shows that the oxidation of DA is an adsorption-controlled process [54].

Analysis of CVs recorded at various concentrations of DA in $100 \mu \mathrm{M}$ UA (in the linear range of 5-80 $\mu \mathrm{M}$ ) and UA in $10 \mu \mathrm{M}$ DA (in the linear range of 50-450 $\mu \mathrm{M}$ ), (Figure $5 \mathrm{e}-\mathrm{h}$ ) demonstrates that the oxidation current of DA and UA increased linearly with concentration. Thus, selective and simultaneous detection of DA and UA was demonstrated. The detection limitation and sensitivity were $6.57 \mu \mathrm{M}$ and $7326.04 \mathrm{nA} \mu \mathrm{M}^{-1}$ $\mathrm{cm}^{-2}$, respectively for DA in the presence of the high concentration $(100 \mu \mathrm{M})$ of UA. Regardless of the effect of the surface area, the sensitivity from the slope (Figure $5 \mathrm{f}$ ) is $260 \mathrm{pA} / \mu \mathrm{M}$. Recently, Xu et al. demonstrated simultaneous detection of DA and neuronal spikes using platinum electrodes in monkey brain [55] and found that a sensitivity of $14 \mathrm{pA} / \mu \mathrm{M}$ was sufficient for this purpose. Hence our electrodes are well above biologically relevant sensitivity limits. Furthermore, this microelectrode can detect the DA peaks in the presence of high concentration UA while others reported the detection of DA using CF based microelectrodes in just PBS solution [56, 57]. As far as we are aware, this is the first time that microelectrode-based CF has been able to detect DA in the presence of high concentrations of UA with good sensitivity and selectivity. The detection limit and sensitivity were $4.53 \mu \mathrm{M}$ and $309.94 \mathrm{nA} \mu \mathrm{M}^{-1} \mathrm{~cm}^{-2}$, respectively, for UA in the presence of $10 \mu \mathrm{M}$ DA.

\section{Conclusion}

We have demonstrated that diamond-coated CF microelectrodes are capable of performing the three main tasks required for closed-loop electric medicine devices: neural stimulation, neural recording, and chemical biosensing. Our previous work has shown that diamond materials are exceptionally long lasting and electrochemically robust, in vivo. However, the mechanical stiffness of bulk diamond films is likely incompatible with the soft, elastic tissues of the nervous system. By incorporating microscale films of diamond on microscale $\mathrm{CF}$ electrodes, the electrochemical benefits of diamond can be combined with the mechanical compliance and micron size of CFs. The electrodes described here are ideal for inclusion in implantable devices for treatment of chronic, long-term medical conditions given their multifunctionality, chemical stability, and small physical size. Such electrodes are highly likely to be a necessary inclusion in the next generation of electric medicine devices, neuroprosthetics and implantable biomonitors to both understand and treat chronic, drug-resistant disorders of the nervous system.

\section{Acknowledgments}

This research was funded by a Project Grant from The National Health and Medical Research Council of Australia (GNT1101717). JF would like to thank the support from the Australian Research Council (ARC) through the ARC Centre of Excellence for Electromaterials Science (CE140100012). The authors acknowledge the use of the Advanced Microscopy Facility at Bio21 (The University of Melbourne) for SEM imaging and the National Vision Research Institute for use of electrophysiology equipment. The authors also acknowledge the facilities, and the scientific and technical assistance of the RMIT Microscopy \& Microanalysis Facility (RMMF), a linked laboratory of Microscopy Australia. The work was performed in part at the Melbourne Centre for Nanofabrication (MCN) in the Victorian Node of the Australian National Fabrication Facility (ANFF). DJG is supported by NHMRC Project Grant GNT1101717 and by an ANFF/MCN Technology Ambassador Fellowship. MM is supported by the Melbourne Neuroscience Institute Fellowship. SP is a shareholder of iBIONICS, a company developing a diamond based retinal implant. SP, 
MM, DJG, and NVA are shareholders and public officers of Carbon Cybernetics Pty Ltd, a company developing diamond and carbon-based medical device components. The other authors declare no conflict of interest.

The raw/processed data required to reproduce these findings cannot be shared at this time as the data also forms part of an ongoing study.

\section{Reference}

1. Loeb, G.E., Neural Prosthetics: A Review of Empirical vs. Systems Engineering Strategies. Applied Bionics and Biomechanics, 2018.

2. Thompson, C.H., et al., Regenerative Electrode Interfaces for Neural Prostheses. Tissue Engineering Part BReviews, 2016. 22(2): p. 125-135.

3. Fattahi, P., et al., A Review of Organic and Inorganic Biomaterials for Neural Interfaces. Advanced Materials, 2014. 26(12): p. 1846-1885.

4. Kozai, T.D.Y., et al., Mechanical failure modes of chronically implanted planar silicon-based neural probes for laminar recording. Biomaterials, 2015. 37: p. 25-39.

5. Karumbaiah, L., et al., Relationship between intracortical electrode design and chronic recording function. Biomaterials, 2013. 34(33): p. 8061-8074.

6. Huffman, M.L. and B.J. Venton, Electrochemical Properties of Different Carbon-Fiber Microelectrodes Using Fast-Scan Cyclic Voltammetry. Electroanalysis, 2008. 20(22): p. 2422-2428.

7. McKenzie, J.L., et al., Decreased functions of astrocytes on carbon nanofiber materials. Biomaterials, 2004. 25(7-8): p. 1309-1317.

8. Huffman, M.L. and B.J. Venton, Carbon-fiber microelectrodes for in vivo applications. Analyst, 2009. 134(1): p. 18-24.

9. Kozai, T.D.Y., et al., Ultrasmall implantable composite microelectrodes with bioactive surfaces for chronic neural interfaces. Nature Materials, 2012. 11(12): p. 1065-1073.

10. Rees, H.R., et al., Carbon Nanopipette Electrodes for Dopamine Detection in Drosophila. Analytical Chemistry, 2015. 87(7): p. 3849-3855.

11. Zachek, M.K., et al., Electrochemical dopamine detection: Comparing gold and carbon fiber microelectrodes using background subtracted fast scan cyclic voltammetry. Journal of Electroanalytical Chemistry, 2008. 614(12): p. 113-120.

12. Nick, C., et al., Three-Dimensional Carbon Nanotube Electrodes for Extracellular Recording of Cardiac Myocytes. Biointerphases, 2012. 7(1-4).

13. Vara, H. and J.E. Collazos-Castro, Biofunctionalized Conducting Polymer/Carbon Microfiber Electrodes for Ultrasensitive Neural Recordings. Acs Applied Materials \& Interfaces, 2015. 7(48): p. 27016-27026.

14. Patel, P.R., et al., Chronic in vivo stability assessment of carbon fiber microelectrode arrays. Journal of Neural Engineering, 2016. 13(6).

15. Wen, Y.P. and J.K. Xu, Scientific Importance of Water-Processable PEDOT-PSS and Preparation, Challenge and New Application in Sensors of Its Film Electrode: A Review. Journal of Polymer Science Part a-Polymer Chemistry, 2017. 55(7): p. 1121-1150.

16. Tang, R.Y., et al., Fabrication of strongly adherent platinum black coatings on microelectrodes array. Science China-Information Sciences, 2014. 57(4).

17. Hadjinicolaou, A.E., et al., Electrical stimulation of retinal ganglion cells with diamond and the development of an all diamond retinal prosthesis. Biomaterials, 2012. 33(24): p. 5812-5820.

18. Bennet, K.E., et al., A Diamond-Based Electrode for Detection of Neurochemicals in the Human Brain. Frontiers in Human Neuroscience, 2016. 10.

19. Bennet, K.E., et al., Development of Conductive Boron-Doped Diamond Electrode: A microscopic, Spectroscopic, and Voltammetric Study. Materials, 2013. 6(12): p. 5726-5741.

20. Almeida, E.C., et al., Electrochemical characterization of doped diamond-coated carbon fibers at different boron concentrations. Thin Solid Films, 2005. 485(1-2): p. 241-246.

21. Garrett, D.J., et al., Diamond for neural interfacing: A review. Carbon, 2016. 102: p. 437-454. 
22. Shalini, J., et al., Ultra-nanocrystalline diamond nanowires with enhanced electrochemical properties. Electrochimica Acta, 2013. 92: p. 9-19.

23. Garrett, D.J., et al., Ultra-nanocrystalline diamond electrodes: optimization towards neural stimulation applications. Journal of Neural Engineering, 2012. 9(1).

24. Birrell, J., et al., Bonding structure in nitrogen doped ultrananocrystalline diamond. Journal of Applied Physics, 2003. 93(9): p. 5606-5612.

25. Tong, W., et al., Optimizing growth and post treatment of diamond for high capacitance neural interfaces. Biomaterials, 2016. 104: p. 32-42.

26. Garrett, D.J., et al., In vivo biocompatibility of boron doped and nitrogen included conductive-diamond for use in medical implants. Journal of Biomedical Materials Research Part B-Applied Biomaterials, 2016. 104(1): $p$. 19-26.

27. Shimoni, O., et al., Development of a Templated Approach to Fabricate Diamond Patterns on Various Substrates. Acs Applied Materials \& Interfaces, 2014. 6(11): p. 8894-8902.

28. Stacey, A., et al., Evidence for Primal sp(2) Defects at the Diamond Surface: Candidates for Electron Trapping and Noise Sources. Advanced Materials Interfaces, 2019. 6(3).

29. Chandra, S., N. Barola, and D. Bahadur, Impedimetric biosensor for early detection of cervical cancer. Chemical Communications, 2011. 47(40): p. 11258-11260.

30. Chaure, F.J., H.G. Rey, and R.Q. Quiroga, A novel and fully automatic spike-sorting implementation with variable number of features. Journal of Neurophysiology, 2018. 120(4): p. 1859-1871.

31. Kozai, T.D.Y., et al., Chronic In Vivo Evaluation of PEDOT/CNT for Stable Neural Recordings. leee Transactions on Biomedical Engineering, 2016. 63(1): p. 111-119.

32. Ibbotson, M.R. and R.F. Mark, Orientation and spatiotemporal tuning of cells in the primary visual cortex of an Australian marsupial, the wallaby Macropus eugenii. Journal of Comparative Physiology a-Neuroethology Sensory Neural and Behavioral Physiology, 2003. 189(2): p. 115-123.

33. Vidyasagar, T.R., et al., Cytoarchitecture and Visual-Field Representation in Area 17 of the Tammar Wallaby (Macropus-Eugenii). Journal of Comparative Neurology, 1992. 325(2): p. 291-300.

34. Ibbotson, M.R., et al., Torsional eye movements during psychophysical testing with rotating patterns. Experimental Brain Research, 2005. 160(2): p. 264-267.

35. Ting, J. and M.L. Lake, Diamond-Coated Carbon-Fiber. Journal of Materials Research, 1994. 9(3): p. 636-642.

36. Ferreira, N.G., L.L.G. Silva, and E.J. Corat, Electrochemical activity of boron-doped diamond electrodes grown on carbon fiber cloths. Diamond and Related Materials, 2002. 11(3-6): p. 657-661.

37. Garrett, D.J., et al., Robust Forests of Vertically Aligned Carbon Nanotubes Chemically Assembled on Carbon Substrates. Langmuir, 2010. 26(3): p. 1848-1854.

38. Butler, J.E., et al., Understanding the chemical vapor deposition of diamond: recent progress. Journal of Physics-Condensed Matter, 2009. 21(36).

39. Wu, Y.H., T. Yu, and Z.X. Shen, Two-dimensional carbon nanostructures: Fundamental properties, synthesis, characterization, and potential applications. Journal of Applied Physics, 2010. 108(7).

40. Mangolini, F., J.B. McClimon, and R.W. Carpick, Quantitative Evaluation of the Carbon Hybridization State by Near Edge X-ray Absorption Fine Structure Spectroscopy. Analytical Chemistry, 2016. 88(5): p. 2817-2824.

41. L.L. Zhang, Q.Y., Y. Tanga, L. Yang, C. Zhanga, Y. Hub, X. Cui, Synthesis and characterization of boron incorporated diamond-like carbon thin films. Thin Solid Films, 2015. 589: p. 457-464.

42. Deslandes, A., et al., Deuterium retention and near-surface modification of ion-irradiated diamond exposed to fusion-relevant plasma. Nuclear Fusion, 2014. 54(7).

43. Smedley, J., et al., Laser patterning of diamond. Part II. Surface nondiamond carbon formation and its removal. Journal of Applied Physics, 2009. 105(12).

44. Ohmagari, S., et al., Near-Edge X-Ray Absorption Fine Structure of Ultrananocrystalline Diamond/Hydrogenated Amorphous Carbon Films Prepared by Pulsed Laser Deposition. Journal of Nanomaterials, 2009.

45. Apollo, N.V., et al., Soft, Flexible Freestanding Neural Stimulation and Recording Electrodes Fabricated from Reduced Graphene Oxide. Advanced Functional Materials, 2015. 25(23): p. 3551-3559.

46. Chan, H.Y., et al., A Novel Diamond Microprobe for Neuro-Chemical and -Electrical Recording in Neural Prosthesis. Journal of Microelectromechanical Systems, 2009. 18(3): p. 511-521. 
47. Patan, M., T. Shah, and M. Sahin, Charge injection capacity of TiN electrodes for an extended voltage range. 2006 28th Annual International Conference of the leee Engineering in Medicine and Biology Society, Vols 115, 2006: p. 5516-+.

48. Harris, A.R., et al., Measuring the effective area and charge density of platinum electrodes for bionic devices. Journal of Neural Engineering, 2018. 15(4).

49. Weitz, A.C., et al., Improving the spatial resolution of epiretinal implants by increasing stimulus pulse duration. Science Translational Medicine, 2015. 7(318).

50. Wong, Y.T., et al., Feasibility of Nitrogen Doped Ultrananocrystalline Diamond Microelectrodes for Electrophysiological Recording From Neural Tissue. Frontiers in Bioengineering and Biotechnology, 2018. 6.

51. Etemadi, L., et al., Embedded Ultrathin Cluster Electrodes for Long-Term Recordings in Deep Brain Centers. Plos One, 2016. 11(5).

52. Fiath, R., et al., Large-scale recording of thalamocortical circuits: in vivo electrophysiology with the twodimensional electronic depth control silicon probe. Journal of Neurophysiology, 2016. 116(5): p. 2312-2330.

53. Komarova, N.S., et al., Enhancement of the Carbon Nanowall Film Capacitance. Electron Transfer Kinetics on Functionalized Surfaces. Langmuir, 2015. 31(25): p. 7129-7137.

54. Fang, J., et al., Co-deposition of carbon dots and reduced graphene oxide nanosheets on carbon-fiber microelectrode surface for selective detection of dopamine. Applied Surface Science, 2017. 412: p. 131-137.

55. Xu, S.W., et al., An integrated system for synchronous detection of neuron spikes and dopamine activities in the striatum of Parkinson monkey brain. Journal of Neuroscience Methods, 2018. 304: p. 83-91.

56. Puthongkham, P., C. Yang, and B.J. Venton, Carbon Nanohorn-modified Carbon Fiber Microelectrodes for Dopamine Detection. Electroanalysis, 2018. 30(6): p. 1073-1081.

57. Dénes Budai, I.H., Beatrix Mészáros, Zsolt K. Bali, Károly Gulya, Electrochemical responses of carbon fiber microelectrodes to dopamine in vitro and in vivo Acta Biologica Szegediensis 2010. 54(2): p. 155-160. 